\title{
Mechanistic insight into the function of the microbiome in lung diseases
}

\author{
Niki D.J. Ubags and Benjamin J. Marsland \\ Affiliation: Faculty of Biology and Medicine, University of Lausanne, Service de Pneumologie, CHUV, \\ Lausanne, Switzerland.
}

Correspondence: Niki D.J. Ubags, Service de Pneumologie, CLE D02-207, Chemin des Boveresses 155, 1066 Epalinges, Switzerland. E-mail: Niki.ubagsđachuv.ch

@ERSpublications

Host-microbe interactions are key determinants of early onset and adult lung diseases http://ow.ly/cuDG30dZg19

Cite this article as: Ubags NDJ, Marsland BJ. Mechanistic insight into the function of the microbiome in lung diseases. Eur Respir J 2017; 50: 1602467 [https://doi.org/10.1183/13993003.02467-2016].

ABSTRACT The lung harbours a diverse array of microbes whose dynamic composition is influenced by both host and environmental factors. Thus far, most studies have described the microbial composition of healthy or diseased lungs and provided an overview of the differences between topographical locations within the respiratory tract. However, insight into the functional mechanisms underlying host-microbe interactions and how they might drive lung health and disease are limited. This review provides an overview of the current mechanistic understanding of the microbiome, crosstalk between tissue compartments, and its involvement in respiratory diseases. 


\section{Introduction}

With each breath the lung is exposed to an array of environmental pollutants, particulates, and both pathogenic and non-pathogenic microbes. Nevertheless, the airways have historically been regarded as a "sterile environment". Over the past few years multiple studies, using culture independent molecular methods, have contributed to defining various lung microbial communities in healthy individuals. Proteobacteria, Firmicutes and Bacteroidetes are the most prevalent phyla in the human respiratory tract, as determined from bronchoalveolar lavage samples of healthy adults [1, 2]. Within these three phyla, Pseudomonas and Haemophilus (Proteobacteria), Streptococcus and Veilonella (Firmicutes), and Prevotella and Porphyromonas (Bacteriodetes) are present at a high frequency in the airways of healthy individuals at the genus level, along with Fusobacterium (Fusobacteria) [1-4].

There are several challenges in assessing the lung microbiome. One of them is the low density of bacterial communities, especially in healthy individuals $\left(10^{2}-10^{3}\right.$ bacteria per $\left.\mathrm{mL}\right)[5,6]$. In addition, there is a risk of cross-contamination of lower respiratory tract samples due to carryover from the upper respiratory tract during sampling. Microbial communities found in the lower respiratory tract of healthy adults closely resemble the community structures found in the oropharynx compared to any other competing source community, such as the nasopharynx or inhaled air $[2,4,7,8]$. However, their relative abundance in bronchoalveolar lavage (BAL) samples generally differs from that in oral samples $[4,5]$ suggestive of a selection process.

The development of the airway microbiome in infants and the exact timing thereof remains unknown, partially due to the difficulty of acquiring lower respiratory tract samples from a healthy cohort. It is traditionally believed that the in utero environment is physiologically sterile. Although further studies are required, there may be a low level of bacterial colonisation during gestation, since a bacterial presence has been detected in cord blood samples from babies delivered via caesarean section [9], in meconium from preterm babies [10] and in the placenta [11]. The establishment of the lung microbiome in early life could be affected by several postnatal factors, after which time the established lung microbiome is subject to selective pressures throughout life represented by environmental and life-style factors. In this review, we will summarise the current understanding of lung microbiome development and function, its involvement in lung health and disease, and thereby highlighting important research questions that require further investigation.

\section{Development of the airway microbiome and implications for the immune system}

The upper airway microbiome composition in healthy infants has been associated with an increased abundance of Haemophilus, Streptococcus and Moraxella, and a decreased abundance of Alloiococcus and Corynebacterium [12]. Most studies determining the development of the airway microbiome in the first year of life have used nasopharyngeal samples. Although the nasopharynx is proposed to be a reservoir for microbes associated with acute respiratory infections, its microbial composition mostly resembles the upper airways [13], and thus cannot be used as a proxy for the lower airways. The infant upper respiratory tract microbiome can be affected by several post-natal factors (table 1) including mode of delivery [14, 15], breast feeding versus formula feeding [16] and the use of antibiotics [12]. Moreover, it is subject to direct or indirect changes induced by environmental and lifestyle factors, such as diet [17, 18]. The use of probiotics has been shown to affect the lung immune response following allergic airway inflammation [1921]; however, whether this affects the lung microbiome remains to be determined. Indeed, there remains a need for investigations into the mechanisms of action of probiotics with a putative benefit for respiratory disease. Investigations determining the lower respiratory tract microbiome in healthy infants shortly after birth and during the first years of life are currently limited due to ethical and technical limitations of obtaining samples. Data is, however, available from mouse studies. The mouse lung microbiome develops within the first days of life, after which time the microbial load and diversity in the lung increases. The lower airways of mice are predominantly colonised with Firmicutes and Proteobacteria in the first days after birth, followed by an increase in the Bacteroidetes phylum prior to weaning (14-21 days old) and through to adulthood [22]. Studies utilising germ-free mice (mice with a complete absence of any microbiota), have shown that the microbiome is essential for development and education of host immune responses, particularly within the context of allergy [23]. Specifically, the absence of a microbiome in germ-free mice results in their exaggerated responsiveness to model allergens, which can be normalised if germ-free mice are recolonised with the commensal gut microbiota from specific pathogen free mice. A recent report by Rемот et al. [24] showed that airway exposure of neonatal mice to specific strains of bacteria, originally isolated from the airways, could either protect or enhance susceptibility of the mice to airway inflammation. These data lead to the enticing hypothesis that exposure to certain microbes early in life could be key for determining the trajectory of an individual towards or away from disease.

Alveolar macrophages are central for pulmonary host defence, and granulocyte-macrophage colony stimulating factor-driven differentiation of pulmonary fetal monocytes to alveolar macrophages [25], may, 
TABLE 1 Summary of factors affecting healthy lung microbiome composition in early and adult life

\begin{tabular}{|c|c|c|c|}
\hline Influencing factor & Sample type & Age at sampling & Changes in airway microbial composition \\
\hline $\begin{array}{l}\text { Delivery mode (vaginal birth } \\
\text { versus caesarean section) }\end{array}$ & Tracheal aspirates [15] & $\begin{array}{l}\text { Within } 6 \mathrm{~h} \text { after } \\
\text { birth }\end{array}$ & $\begin{array}{l}\text { Vaginal birth: no alterations in lower airway } \\
\text { microbiome. } \\
\text { Caesarean section: no alterations in lower } \\
\text { airway microbiome. }\end{array}$ \\
\hline $\begin{array}{l}\text { Feeding choices } \\
\text { (breastfeeding versus } \\
\text { formula feeding) }\end{array}$ & Nasopharyngeal swab [16] & 6 weeks old & $\begin{array}{l}\text { Breastfeeding versus formula feeding: } \\
\text { Increased presence and abundance of } \\
\text { Dolosigranulum and Corynebacterium } \\
\text { Decreased abundance of Staphylococcus, } \\
\text { Prevotella and Veilonella }\end{array}$ \\
\hline Diet composition (fibre intake) & $\begin{array}{l}\text { Murine lung tissue }[17,18] \text { (no data on } \\
\text { human lung microbiome composition } \\
\text { available) }\end{array}$ & Adult & $\begin{array}{l}\text { High fibre diet: altered Firmicutes to } \\
\text { Bacteroidetes ratio }\end{array}$ \\
\hline Probiotics & & & $\begin{array}{l}\text { Probiotics use: no data available on airway } \\
\text { microbiome composition }\end{array}$ \\
\hline
\end{tabular}

at least in part, be due to early colonisation in the airways. Indeed, maturation of alveolar macrophages, and other pulmonary $\mathrm{CD} 11 \mathrm{c}^{+}$cells, has been shown to be initiated by airway exposure of newborn mice to a mixture of microbial extracts [26]. Neonatal gut microbial exposure plays an important role in invariant natural killer $\mathrm{T}$ (iNKT) cell accumulation in the lung. In early-life, but not adult life, re-colonisation of germ-free mice with a conventional gut microbiota abrogated iNKT cell accumulation in the lung by induction of CXCL16, consequently protecting mice against exaggerated allergic airway inflammation [27]. The frequency of $\mathrm{CD}_{11} \mathrm{~b}^{+}$conventional dendritic cells is increased in the mouse lung during the first 2 weeks of life, and microbial colonisation promotes induction of regulatory $\mathrm{T}$ (Treg) cells via transient expression of programmed death ligand-1 (PD-L1) on these CD11 ${ }^{+}$dendritic cells [22]. In addition, blockade of PD-L1 during the first 2 weeks of life led to exaggerated allergic airway inflammation after house dust mite extract exposure, which was maintained through to adulthood [22]. There has been an indication that microbial exposure occurs in utero; however, whether this represents actual colonisation and formation of a stable microbiome requires further investigation. However, there is evidence that microbial products are found in amniotic fluid [28], and KRAMER et al. [29] reported that intra-amniotic endotoxin exposure accelerated monocyte maturation and differentiation into macrophage-like cells in the preterm fetal sheep lung.

Early life exposure to microbes and their products thus appears important in long-term lung health, and it has been demonstrated that the murine "window of opportunity" lies within the first 2-3 weeks of life, during which a decreased responsiveness towards allergens develops. Although there are considerable technical and ethical limitations in sampling the lower respiratory tract of babies, the current molecular insights gathered from animal studies should be confirmed in a clinical setting in order to advance towards possible therapeutic or diagnostic strategies.

\section{Gut-lung crosstalk}

Analysis of the "normal" microbiome in healthy individuals has revealed profound differences in microbiota composition between various body compartments, highlighting the tissue habitat as a key determinant of colonisation. Over the past years the importance of microbiome crosstalk between these different compartments of the human body in health and disease has been increasingly recognised. Respiratory diseases have not only been associated with microbial dysbiosis in the lung, but also the gut microbiome has been linked with lung health and disease (figure 1).

It has become increasingly evident that not only can intestinal disorders have pulmonary manifestations [30], but also vice versa. Respiratory diseases, including asthma, chronic obstructive pulmonary disease 


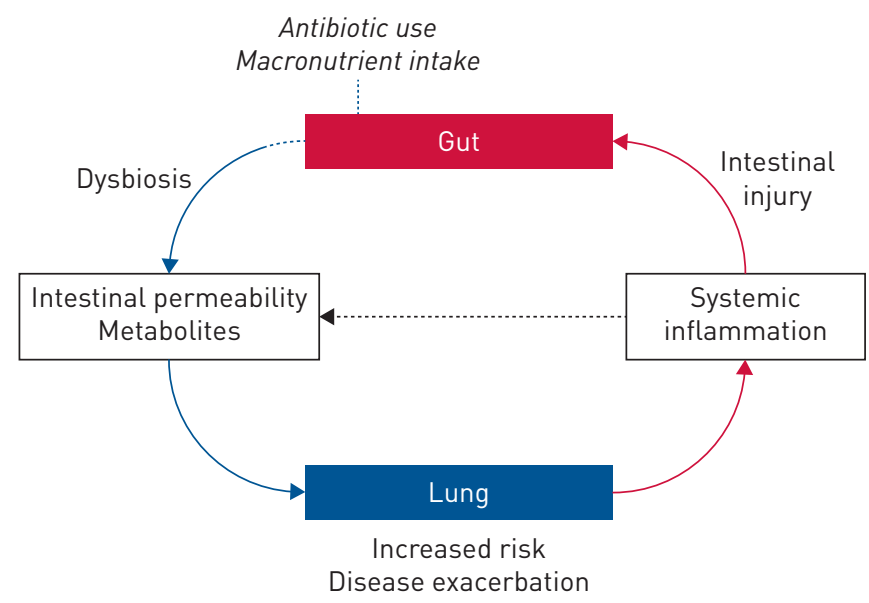

FIGURE 1 Gut-lung crosstalk in respiratory diseases. Respiratory diseases lasthma, chronic obstructive pulmonary disease, cystic fibrosis and viral infections) are accompanied by a component of intestinal disease manifestation, which may be driven by systemic inflammation. Respiratory influenza infection can induce intestinal injury, which is mediated by lymphocyte migration from the respiratory tract into the intestinal mucosa [33]. Alterations in gut microbial composition (dysbiosis) induced by antibiotic use or alterations in macronutrient intake (e.g. low fiber or high fat intake) can increase intestinal permeability and alter metabolite production in the gut, which can consequently lead to an increased risk for respiratory diseases, or exacerbation of pre-existing lung disease. Translocation of gut bacteria into the lung has been observed in critical illness, such as sepsis, acute respiratory distress syndrome or post-stroke infection [41, 42]. This is thought to be mediated by compromised barrier integrity.

(COPD), cystic fibrosis and viral respiratory infection are accompanied by a component of intestinal disease manifestation [30-35]. On the other hand it has been demonstrated that low intestinal microbial diversity during infancy is correlated with an asthmatic phenotype during childhood [36], suggesting a role for the gut microbiome in the onset of asthma. One caveat to the common generalisation that gut microbial diversity is positively linked with health is the observation that breastfed children have a lower diversity gut microbiota to that of formula fed babies [37], while breast feeding is considered to lower the risk of allergy [38, 39]. Thus, one could extrapolate that an intestinal microbiota with an over-representation of beneficial microbes might be sufficient for health, and a highly diverse microbiota could reflect a "dilution" of potentially detrimental pathobionts.

WANG et al. [33] demonstrated that intestinal injury following respiratory influenza infection was not caused by influenza infection of the intestine directly, since no virus was detected in the small intestine following intranasal infection, and intra-gastric administration of the virus directly into the intestine did not lead to intestinal immune injury. In fact, the injury was shown to be mediated by lymphocytes migrating from the respiratory tract into the intestinal mucosa during infection via the CCL25-CCR9 axis [33].

Profound differences in the intestinal microbiota of cystic fibrosis patients were detected; however, these changes have not yet been linked to severity of the disease. Moreover, it is well known that dysfunction of the CFTR gene itself contributes to poor digestive functions due to improper neutralisation of gastric bile, which in turn changes the ecological environment in the gut [40]. Interestingly, a recent study demonstrated that bacteria from the gastrointestinal tract can translocate to the lungs in patients with sepsis and acute respiratory distress syndrome, which is likely mediated by compromised barrier integrity [41]. Moreover, post-stroke infection in patients is most likely caused by dissemination of selective strains of bacteria originating from the gut microbiota; indeed, a source of the bacteria in the lungs of post-stroke mice has been shown to be the small intestine [42]. These studies suggest an important role for the gut -lung axis in critical illness and highlight the need for intestinal barrier integrity.

\section{Antibiotic use}

Antibiotic induced alterations in the gut microbiota during early life have been shown to exacerbate experimental allergic airway disease following allergen exposure in adult life [43-45]. In an ovalbumin model of Th2 induced allergic airway inflammation, neonatal, but not adult, vancomycin treatment was shown to exacerbate the allergic inflammatory response in the lung [43]. Vancomycin treatment did not reduce the bacterial load in the gut, but resulted in a profound shift in gut microbial composition, characterised by an almost complete depletion of Bacteroidetes which were replaced by an overgrowth of Lactobacilli. Immunologically, this correlated with a decrease in intestinal Treg cells [43]. Interestingly, 
streptomycin was shown to exacerbate the Th1/Th17-driven inflammatory lung disease, hypersensitivity pneumonitis, whereas vancomycin did not exacerbate this type of disease [44]. Although there were only subtle shifts in gut microbial composition following streptomycin treatment, Bacteroidetes, the most abundant phylum in the intestine of streptomycin-treated mice, demonstrated the strongest association with hypersensitivity pneumonitis severity [44]. These studies demonstrate that changes in intestinal microbial composition can have profound consequences in immunologically distinct disease models. Interestingly, depletion of gut microbiota in mice by broad-spectrum antibiotic treatment (consisting of ampicillin, neomycin, metronidazole and vancomycin) has been shown to impair pulmonary host defence against Streptococcus pneumoniae infection, which was likely driven by gut microbiota-mediated alterations in the metabolic status of alveolar macrophages [46].

\section{Dietary composition}

Dietary fibre intake has been shown to alter gut and lung microbial composition by shifting the Firmicutes to Bacteroidetes ratio [17, 18]. Consumption of a high fibre diet, which increases short chain fatty acid (SCFA) levels in the circulation and the gut, or direct administration of SCFAs, protects mice against the development of asthma $[17,18]$. This protection was mediated by changes in dendritic cell haematopoiesis and function, arguing for a gut-bone marrow-lung axis [18]. In line with these results, intestinal helminth infection has been reported to alter gut microbial composition, thereby increasing SCFA production, which consequently led to attenuation of house dust mite-induced allergic airway inflammation [47]. Notably, an increased intake of dietary fat leads to the opposite alterations in the Firmicutes to Bacteroidetes ratio [48] in the gut and SCFA levels [49] in the circulation. Whether these alterations affect lung microbiome composition and the pulmonary immune response has yet to be fully delineated, but is clearly an important avenue for further research since obesity is a risk factor for the development of both atopic and non-atopic asthma [50].

\section{Influence of the microbiome on development and progression of pulmonary disease}

Colonisation of the lungs in early life is a highly dynamic process and can be influenced by external factors, thereby increasing the susceptibility for developing early onset lung disease. Moreover, lung microbial composition can be influenced by environmental exposures and lifestyle factors consequently resulting in an enhanced susceptibility to develop pulmonary disease in adulthood. These factors can also contribute to disease exacerbations and chronicity. Here we will discuss how the bacterial lung microbiome can affect development and progression of both early onset and adult lung diseases (figure 2).

\section{Asthma}

The presence of microbes has been demonstrated to be a key player in asthma development, since studies in germ-free mice show that a lack of microbial colonisation before allergen exposure increases allergic airway inflammation and that re-colonisation is able to rescue this phenotype [23]. Early life microbial exposure is important for tolerance induction to allergens later in life, and can be altered by postnatal use of antibiotics [43]. STEIN et al. [51] recently reported that a microbial rich indoor environment, as seen in the Amish population and characterised by high endotoxin levels, protects children against asthma development. In addition, it was demonstrated that expression of genes involved in innate immune responses to microbes were upregulated on peripheral blood leukocytes from these children. Moreover, exposure of mice to dust extracts collected from the indoor environment in Amish homes attenuated ovalbumin-induced airway hyperresponsiveness. This protective effect was shown to be dependent on MyD88-Trif signalling [51]. Taken together, these data suggest that innate immune stimulation in children may be crucial in protection against asthma development.

The first studies that characterised the airway microbiome in asthmatic patients reported an increased abundance of Proteobacteria, whereas the Bacteroidetes phylum was under-represented compared to healthy control [2,52]. Inhaled corticosteroid treatment in asthmatic patients showed increased airway bacterial diversity, which correlated with increased airway hyperresponsiveness [52] and airflow obstruction [53]: another caveat to the concept that microbial diversity is always beneficial. Furthermore, it has been suggested that Haemophilus parainfluenzae, which was suggested to be expanded in the airways of a subset of patients with corticosteroid-resistant asthma, suppresses the alveolar macrophage response to corticosteroids by increased TAK1/MAPK activation [6]. However, whether this proposed mechanism of action can be extrapolated to other bacterial species involved in corticosteroid-resistance remains to be elucidated.

Although several early life and environmental factors have been related to alterations in airway colonisation and asthma development, our understanding of the relationship between airway microbial composition and asthma severity remains under-investigated. Recent phenotypic classifications of asthma 
a)

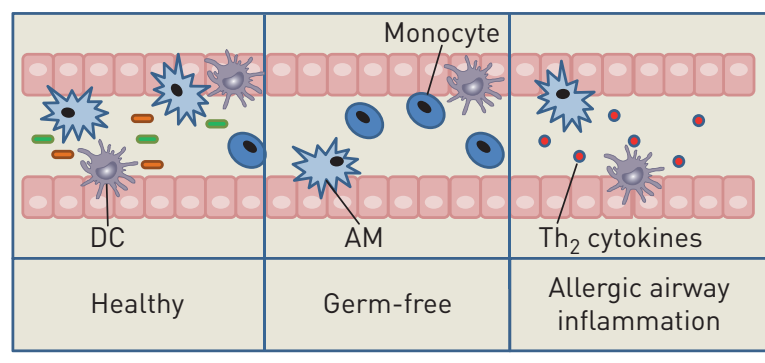

c)

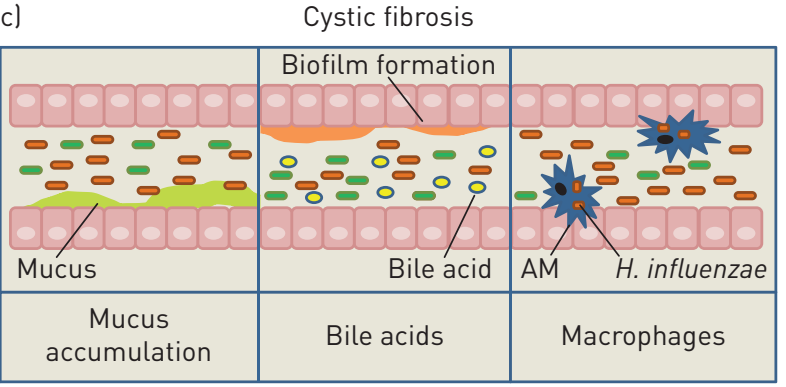

b)

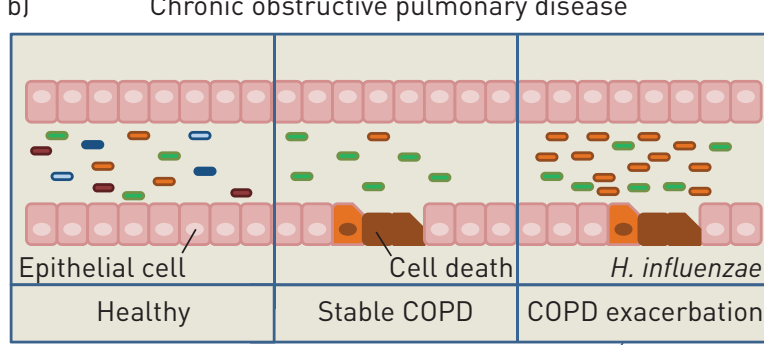

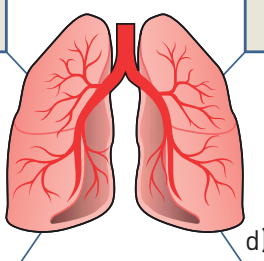

d) Lung transplantation

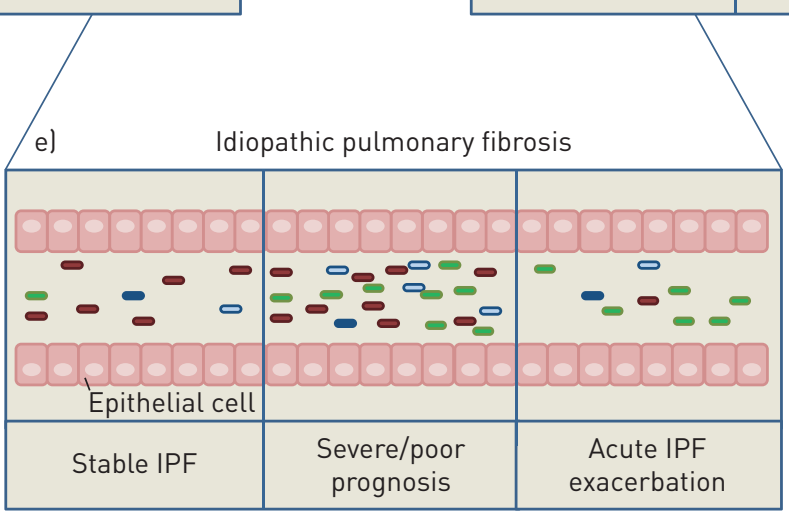

FIGURE 2 Host-microbiome interactions in respiratory diseases. Host-microbiome interactions play a fundamental role in the pathogenesis of a variety of respiratory diseases. a) The presence of microbes is important in the development of asthma. A lack of microbial colonisation (germ-free environment) is characterised by delayed differentiation and maturation from monocytes to alveolar macrophages in early life, and a decreased presence of dendritic cells and alveolar macrophages (AM) in adulthood. Induction of allergic airway inflammation in germ-free mice leads to exaggerated allergic inflammation, which is likely mediated by delayed maturation of pulmonary immune cells [23]. b) Microbial diversity is decreased in the airway of stable chronic obstructive pulmonary disease (COPD) patients. An acute COPD exacerbation can lead to microbial expansion of bacterial species (such as Haemophilus influenza, here depicted in orange) which were already present in low abundance in the lung during steady state COPD [59]. c) The cystic fibrosis lung is characterised by impaired mucociliary clearance leading to mucus accumulation, thereby creating an ideal environment for growth and expansion of certain types of bacteria. Aspiration is a common symptom in patients with cystic fibrosis (and other chronic lung diseases) and can increase bile acid levels in the lung. Enhanced levels of bile acids can lead to biofilm formation and consequently increase antibiotic tolerance, which can lead to further microbial expansion [74]. Furthermore, CFTR deficient macrophages can effectively ingest Pseudomonas aeruginosa (here depicted in orange). However, these macrophages have limited capacity to kill this microbe, which can consequently lead to bacterial outgrowth [64]. d) The microbial composition in the lung allograft originates from various microbiome sources. The immunological profile of alveolar macrophages from lung transplant patients have been linked to specific microbial signatures (pro-inflammatory (in red), intermediate (in blue) and pro-remodelling (in green)). The pro-inflammatory profile was linked to dysbiosis of Firmicutes and Proteobacteria, whereas a Bacteroidetes-driven dysbiosis was linked to the pro-remodelling activation profile. The intermediate profile reflected a more balanced environment [72]. e) Bacterial load is increased in patients with idiopathic pulmonary fibrosis (IPF) with severe disease and poor prognosis compared to stable disease. Microbial diversity is decreased during acute IPF exacerbation, whereas total bacterial load and abundance of Proteobacteria (in green) is increased.

have contributed to improved treatment regimens for asthmatics [54]. However, whether these phenotypes can be linked to a distinct microbial composition in the respiratory tract remains to be determined.

\section{Chronic obstructive pulmonary disease}

COPD is a lung disease characterised by a largely irreversible chronic obstruction of airflow that interferes with normal breathing. The course of the disease is characterised by intermittent exacerbations [55]. 
Moraxella, Haemophilus and Actinobacteria are over-represented in the lungs of COPD patients [1, 2]. Loss of microbial diversity has been observed mostly in COPD patients with severe impairment of lung function and during exacerbations, which was particularly associated with dominance of Pseudomonas spp. [1, 56, 57]. Of note, higher abundance of Moraxella was found in COPD patients with a more neutrophilic exacerbation phenotype [56], suggesting an increased susceptibility to microbiome alterations during acute exacerbations in a specific subset of patients. When looking at host-microbiome interactions, CXCL8/IL-8 levels in sputum were shown to have a significant correlation with specific microbiota groups, suggesting that this may be a biomarker to monitor lung microbial composition [56]. Recent mechanistic insight from a mouse model of COPD supports the important role for the lung microbiota in COPD through the induction of increased IL-17A-mediated inflammatory responses and autoantibody production [58]. Of note, a study by Molyneaux et al. [59] analysed the airway microbiome in induced sputum from COPD patients, asymptomatic smokers and healthy controls before and after induced rhinovirus exacerbation. A significant expansion of $H$. influenza was observed in COPD patients following exacerbation. Interestingly, $H$. influenza was already present in low abundance at baseline in COPD patients [59]. These data suggest that bacterial expansion following exacerbation occurred from an existing bacterial community, rather than by acquisition of new bacterial species.

\section{Cystic fibrosis}

Cystic fibrosis is a multi-organ disease in which pulmonary manifestations of the disease are the leading cause of morbidity and mortality $[60,61]$. The lung environment in cystic fibrosis patients, characterised by depletion of the airway surface liquid layer leading to ciliary dyskinesis and impaired mucociliary clearance, is ideal for microbial colonisation [62]. A small core microbiome has been described in sputum of both paediatric and adult cystic fibrosis patients. Lower lung function and microbial diversity was observed in older patients, which coincided with a higher proportion of samples dominated by Pseudomonas or Burkholderia and a decreased proportion of Streptococcus [63]. CFTR deficiency in macrophages from cystic fibrosis patients was shown to affect the microbicidal function of these phagocytes when exposed to P. aeruginosa, which could, in part, explain the increase in Pseudomonas observed in cystic fibrosis [64]. Furthermore, an increase in S. aureus was observed at a very early age in cystic fibrosis patients [65-68], and increased respiratory colonisation with $S$. aureus is associated with poor respiratory outcome. Decreased co-colonisation with other potential pathogens, including $S$. pneumoniae and $H$. influenzae, has been observed in the presence of $S$. aureus in both healthy infants $[69,70]$ and infants with cystic fibrosis [68]. Interestingly, the abundance of Corynebacteraceae, a potentially beneficial bacterium, was significantly lower in cystic fibrosis patients $[67,68]$. It is suggested that macrophage phenotypes shift during cystic fibrosis pathogenesis [71], however, whether changes in lung microbiome composition could underlie this phenotypic switch, as observed following lung transplantation [72], remains to be investigated.

Exacerbations caused by an acute response to infection underlie most of the lung damage and worsening of disease seen in cystic fibrosis. Treatment with antibiotics before 6 months of age induces a significant shift in microbial composition, thereby increasing gram-negative bacteria [68]. Aggressive antibiotic therapy and chronic colonisation of the adult cystic fibrosis lung contributes to the emergence of antibiotic-resistant organisms including multidrug-resistant $P$. aeruginosa and methicillin-resistant S. aureus [73]. The observed decrease in the primarily highly abundant Corynebacterium and Dolosigranulum following antibiotic treatment $[67,68]$ may have important therapeutic implications, since these genera were also found to be associated with the stability of the respiratory microbiota and a decreased risk for respiratory infections [16]. Interestingly, bile acid (which is increased in cystic fibrosis patient lungs following aspiration) has been shown to enhance $P$. aeruginosa biofilm formation and is able to increase antibiotic tolerance [74], suggesting that analysis of bile acid levels may be a potential indicator of disease prognosis and development. Taken together, these findings point towards an interplay between different bacteria in cystic fibrosis, and suggest that modulating the airway microbiota allowing beneficial microbes to outcompete the pathogens could reduce inflammation and lead to improved respiratory health.

\section{Lung transplantation}

Lung transplantation is an increasingly common option for patients with advanced stage lung disease, of which the majority are suffering from COPD, idiopathic pulmonary fibrosis (IPF) or cystic fibrosis [75]. The transplanted lung offers particular ecological conditions for microbes; of particular note is the long-term use of antibiotics and immunosuppressive therapy that may shape the lung microbiome in transplant recipients. Existing pulmonary infections, such as B. cepacia infection, in cystic fibrosis recipients have been shown to present as a risk factor for increased mortality following transplantation $[76,77]$. Although cystic fibrosis patients usually undergo a bilateral transplant, the upper airways and 
sinuses may still serve as a reservoir for bacteria and can repopulate the donor lung. Richness and diversity is decreased in transplant patients [78], and this decrease in diversity may be tied to the emergence of a predominant organism during periods of infection. A predominance of Firmicutes has been reported in transplant patients who developed bronchiolitis obliterans syndrome (BOS), whereas mainly Proteobacteria were found in the lungs of patients without BOS. Moreover, BOS has been associated with an increased variability of the lung microbiome over time [79]. However, it remains to be elucidated whether particular pre-transplant infections may be associated with poor post-transplantation outcomes.

A recent study offers the first mechanistic insight regarding host-microbiome interactions in the transplanted lung by characterising the immunological profile of primarily alveolar macrophages from BAL fluid of transplant patients and linking this to their respective microbial signature. Three sample groups were delineated based on their cell activation profile: 1) pro-inflammatory, 2) intermediate and 3) pro-remodelling [72]. Inflammation was found to peak at 3-4 months post-transplant followed by an increase in the remodelling activation profile at later time points. The highest prevalence of a balanced microbiota was found in the sample group with an "intermediate" cell activation profile, reflecting homeostasis. Interestingly, dysbiosis linked to Firmicutes and Proteobacteria was primarily associated with an inflammatory gene expression profile, and corresponded to low alveolar macrophage and high neutrophil percentages in bronchoalveolar lavage (BAL) fluid. Comparatively, Bacteroidetes-driven dysbiosis was more frequently associated with a pro-remodelling cell activation profile and high alveolar macrophage and low neutrophil percentages [72]. This suggests that dynamic changes occurring in the airways of patients undergoing lung transplant appear to be reflected in the cell activation profile and microbiota composition. However, whether cell activation profiles at specific time-points post-transplantation may be predictive of the development of BOS or even mortality remains to be determined.

\section{Idiopathic pulmonary fibrosis}

IPF is a chronic, progressive and fatal lung disease. Although the cause of IPF remains controversial, active infection in IPF has been associated with high morbidity and mortality [80]. Viruses may contribute to initiation and progression of the disease [81, 82], and although IPF exacerbations are usually thought to be of non-infectious aetiology, viral infections have the potential to take part in disease exacerbations [83]. Recently our limited understanding of the contribution of bacteria in the onset and progression of the disease has progressed. MolynEAux et al. [84] reported an increased bacterial load and decreased microbial diversity in BAL samples from IPF patients. Interestingly, subjects with high bacterial load at time of IPF diagnosis were at increased risk of mortality compared to subjects with a low bacterial burden. However, no correlation was found between specific microbes and disease progression [84], whereas retrospective analysis of the lung microbiome in BAL samples from the COMET-cohort suggested that presence of specific Streptococcus operational taxonomic units (OTU) or Staphylococcus OTU was associated with worse outcomes of IPF [85]. These data suggest that although there are clear differences in the IPF BAL microbiome (increase in Haemophilus, Neisseria, Streptococcus and Veillonella species) compared to healthy subjects, the bacterial load affects survival in these patients [84]. Acute exacerbations are linked with significant mortality and morbidity in IPF patients, and an increase in BAL bacterial burden has been associated with acute IPF exacerbation and disease state [86]. The abundance of Proteobacteria was increased in patients with acute exacerbation of IPF and within this phylum Stenotrophomonas sp. and Campylobacter sp. were more prominent.

Two recent studies have described host-microbe interactions in IPF patient cohorts by investigating the association between peripheral blood mononuclear cell (PBMC) transcriptomics and lung microbial communities at IPF diagnosis. Molyneaux et al. [87] demonstrated that an altered or more abundant microbiome in IPF patients was linked to over expression of genes involved in host defence responses, which correlated with poor survival. Longitudinal analysis revealed parallel gene expression changes between stable and progressive IPF patients; however, the magnitude of gene expression levels were found to depend on the nature of the disease [87]. Huang et al. [88] demonstrated that immune pathways in PBMCs can be down regulated by microbes, exhibiting increased abundance and decreased community diversity, that are associated with a poorer progression-free survival in those subjects. The outcomes of these studies may pave the path for biomarker development for prognosis and survival prediction in IPF patients. However, since correlations between alterations in gene expression and microbial communities in the lung do not establish causality, it is crucial to perform additional interventional studies in the presence and absence of antimicrobial therapy in which host-microbe interactions are assessed.

\section{Outlook}

Although there have been global efforts to assess the composition of the microbiome in both healthy and diseased lungs, these studies have been mainly descriptive in nature. Therefore, a priority should be to 
further focus on developing a comprehensive understanding of the mechanisms underlying the observed alterations in lung microbial composition and their contribution to the pathogenesis of respiratory disease. Investigating host-microbe interactions will aid in developing this additional mechanistic insight. Furthermore, viruses and fungal components of the microbiota have been described in the respiratory tract $[89,90]$, and have been linked to disease states [91-93]. However, mechanistic studies determining the functional impact of alterations in the virome and mycobiome on lung health and disease are scarce. It is imperative to expand our understanding of the functional impact of viral and fungal constituents in the onset and progression of lung disease. In addition, it will be important to focus on assessment of the involvement of alterations in fungal and viral communities in reshaping of the bacterial microbiome and consequent host responses.

The focus of future mechanistic microbiome studies should be directed towards understanding interactions between the host and microbes, using not only metagenomics, but also metabolomics and metatranscriptomics, which can be integrated using network based approaches. This will allow us to expand our knowledge on which byproducts (metabolites) are formed (metabolomics) and which microbial genes are differentially expressed (metatranscriptomics) during, for example, a disease exacerbation. An important challenge of such an approach is to determine whether a signal is generated by the host or by the microbiome itself. To delineate these specific effects, cellular studies may come into play. Isolation of bacterial strains of interest from the respiratory tract and consequent co-culture with the cells (or matrix) of interest, may be useful to pick apart cellular mechanisms involved in a specific host - microbe interaction. However, caution is recommended when extrapolating results from cellular studies to the clinical situation, since in vitro/ex vivo studies decrease the complexity of the environment such as cell-cell and cell-matrix interactions.

\section{BOX 1 Highlights}

Investigations determining microbiome development of the lower respiratory tract in healthy newborns and infants are crucial to further understand the time-frame at which early onset lung health or disease can be determined.

Host-microbe interactions play a fundamental role in pulmonary immune maturation and development.

Mapping of airway microbial composition linked to discrete clinical phenotypes could enhance treatment strategies.

\section{References}

1 Erb-Downward JR, Thompson DL, Han MK, et al. Analysis of the lung microbiome in the "healthy" smoker and in COPD. PLoS One 2011; 6: e16384.

2 Hilty M, Burke C, Pedro H, et al. Disordered microbial communities in asthmatic airways. PLoS One 2010; 5 e8578.

3 Charlson ES, Bittinger K, Chen J, et al. Assessing bacterial populations in the lung by replicate analysis of samples from the upper and lower respiratory tracts. PLoS One 2012; 7: e42786.

4 Charlson ES, Bittinger K, Haas AR, et al. Topographical continuity of bacterial populations in the healthy human respiratory tract. Am J Respir Crit Care Med 2011; 184: 957-963.

5 Bassis CM, Erb-Downward JR, Dickson RP, et al. Analysis of the upper respiratory tract microbiotas as the source of the lung and gastric microbiotas in healthy individuals. MBio 2015; 6: e00037.

6 Goleva E, Jackson LP, Harris JK, et al. The effects of airway microbiome on corticosteroid responsiveness in asthma. Am J Respir Crit Care Med 2013; 188: 1193-1201.

7 Segal LN, Alekseyenko AV, Clemente JC, et al. Enrichment of lung microbiome with supraglottic taxa is associated with increased pulmonary inflammation. Microbiome 2013; 1: 19.

8 Dickson RP, Erb-Downward JR, Freeman CM, et al. Spatial variation in the healthy human lung microbiome and the adapted island model of lung biogeography. Ann Am Thorac Soc 2015; 12: 821-830.

9 Jimenez E, Fernandez L, Marin ML, et al. Isolation of commensal bacteria from umbilical cord blood of healthy neonates born by cesarean section. Curr Microbiol 2005; 51: 270-274.

10 Jimenez E, Marin ML, Martin R, et al. Is meconium from healthy newborns actually sterile? Res Microbiol 2008; 159: $187-193$.

11 Aagaard K, Ma J, Antony KM, et al. The placenta harbors a unique microbiome. Sci Transl Med 2014; 6: $237 \mathrm{ra} 265$.

12 Teo SM, Mok D, Pham K, et al. The infant nasopharyngeal microbiome impacts severity of lower respiratory infection and risk of asthma development. Cell Host Microbe 2015; 17: 704-715.

13 Marsh RL, Kaestli M, Chang AB, et al. The microbiota in bronchoalveolar lavage from young children with chronic lung disease includes taxa present in both the oropharynx and nasopharynx. Microbiome 2016; 4: 37.

14 Dominguez-Bello MG, Costello EK, Contreras M, et al. Delivery mode shapes the acquisition and structure of the initial microbiota across multiple body habitats in newborns. Proc Natl Acad Sci USA 2010; 107: 11971-11975.

15 Lal CV, Travers C, Aghai ZH, et al. The Airway Microbiome at Birth. Sci Rep 2016; 6: 31023.

16 Biesbroek G, Bosch AA, Wang X, et al. The impact of breastfeeding on nasopharyngeal microbial communities in infants. Am J Respir Crit Care Med 2014; 190: 298-308. 
17 Thorburn AN, McKenzie CI, Shen S, et al. Evidence that asthma is a developmental origin disease influenced by maternal diet and bacterial metabolites. Nat Commun 2015; 6: 7320.

18 Trompette A, Gollwitzer ES, Yadava K, et al. Gut microbiota metabolism of dietary fiber influences allergic airway disease and hematopoiesis. Nat Med 2014; 20: 159-166.

19 Feleszko W, Jaworska J, Rha RD, et al. Probiotic-induced suppression of allergic sensitization and airway inflammation is associated with an increase of T regulatory-dependent mechanisms in a murine model of asthma. Clin Exp Allergy 2007; 37: 498-505.

20 Sagar S, Morgan ME, Chen S, et al. Bifidobacterium breve and Lactobacillus rhamnosus treatment is as effective as budesonide at reducing inflammation in a murine model for chronic asthma. Respir Res 2014; 15: 46.

21 Zuany-Amorim C, Sawicka E, Manlius C, et al. Suppression of airway eosinophilia by killed Mycobacterium vaccae-induced allergen-specific regulatory T-cells. Nat Med 2002; 8: 625-629.

22 Gollwitzer ES, Saglani S, Trompette A, et al. Lung microbiota promotes tolerance to allergens in neonates via PD-L1. Nat Med 2014; 20: 642-647.

23 Herbst T, Sichelstiel A, Schar C, et al. Dysregulation of allergic airway inflammation in the absence of microbial colonization. Am J Respir Crit Care Med 2011; 184: 198-205.

24 Remot A, Descamps D, Noordine M, et al. Bacteria isolated from lung modulate asthma susceptibility in mice. ISME J 2017; 11: 1061-1074.

25 Guilliams M, De Kleer I, Henri S, et al. Alveolar macrophages develop from fetal monocytes that differentiate into long-lived cells in the first week of life via GM-CSF. J Exp Med 2013; 210: 1977-1992.

26 Kasahara K, Matsumura Y, Ui K, et al. Intranasal priming of newborn mice with microbial extracts increases opsonic factors and mature CD11c+ cells in the airway. Am J Physiol Lung Cell Mol Physiol 2012; 303: L834-L843.

27 Olszak T, An D, Zeissig S, et al. Microbial exposure during early life has persistent effects on natural killer $\mathrm{T}$ cell function. Science 2012; 336: 489-493.

28 Collado MC, Rautava S, Aakko J, et al. Human gut colonisation may be initiated in utero by distinct microbial communities in the placenta and amniotic fluid. Sci Rep 2016; 6: 23129.

29 Kramer BW, Joshi SN, Moss TJ, et al. Endotoxin-induced maturation of monocytes in preterm fetal sheep lung. Am J Physiol Lung Cell Mol Physiol 2007; 293: L345-L353.

30 Roussos A, Koursarakos P, Patsopoulos D, et al. Increased prevalence of irritable bowel syndrome in patients with bronchial asthma. Respir Med 2003; 97: 75-79.

31 Baral V, Connett G. Acute intestinal obstruction as a presentation of cystic fibrosis in infancy. J Cyst Fibros 2008 7: 277-279.

32 Keely S, Hansbro PM. Lung-gut cross talk: a potential mechanism for intestinal dysfunction in patients with COPD. Chest 2014; 145: 199-200.

33 Wang $\mathrm{J}$, Li F, Wei $\mathrm{H}$, et al. Respiratory influenza virus infection induces intestinal immune injury via microbiota-mediated Th17 cell-dependent inflammation. J Exp Med 2014; 211: 2397-2410.

34 Dilantika C, Sedyaningsih ER, Kasper MR, et al. Influenza virus infection among pediatric patients reporting diarrhea and influenza-like illness. BMC Infect Dis 2010; 10: 3.

35 Rutten EP, Lenaerts K, Buurman WA, et al. Disturbed intestinal integrity in patients with COPD: effects of activities of daily living. Chest 2014; 145: 245-252.

36 Abrahamsson TR, Jakobsson HE, Andersson AF, et al. Low gut microbiota diversity in early infancy precedes asthma at school age. Clin Exp Allergy 2014; 44: 842-850.

37 Praveen $\mathrm{P}$, Jordan F, Priami C, et al. The role of breast-feeding in infant immune system: a systems perspective on the intestinal microbiome. Microbiome 2015; 3: 41.

38 Verhasselt V, Milcent V, Cazareth J, et al. Breast milk-mediated transfer of an antigen induces tolerance and protection from allergic asthma. Nat Med 2008; 14: 170-175.

39 Kull I, Almqvist C, Lilja G, et al. Breast-feeding reduces the risk of asthma during the first 4 years of life. J Allergy Clin Immunol 2004; 114: 755-760.

40 De Lisle RC, Borowitz D. The cystic fibrosis intestine. Cold Spring Harb Perspect Med 2013; 3: a009753.

41 Dickson RP, Singer BH, Newstead MW, et al. Enrichment of the lung microbiome with gut bacteria in sepsis and the acute respiratory distress syndrome. Nat Microbiol 2016; 1: 16113.

42 Stanley D, Mason LJ, Mackin KE, et al. Translocation and dissemination of commensal bacteria in post-stroke infection. Nat Med 2016; 22: 1277-1284.

43 Russell SL, Gold MJ, Hartmann M, et al. Early life antibiotic-driven changes in microbiota enhance susceptibility to allergic asthma. EMBO Rep 2012; 13: 440-447.

44 Russell SL, Gold MJ, Reynolds LA, et al. Perinatal antibiotic-induced shifts in gut microbiota have differential effects on inflammatory lung diseases. J Allergy Clin Immunol 2015; 135: 100-109.

45 Arrieta MC, Stiemsma LT, Dimitriu PA, et al. Early infancy microbial and metabolic alterations affect risk of childhood asthma. Sci Transl Med 2015; 7: 307ra152.

46 Schuijt TJ, Lankelma JM, Scicluna BP, et al. The gut microbiota plays a protective role in the host defence against pneumococcal pneumonia. Gut 2016; 65: 575-583.

47 Zaiss MM, Rapin A, Lebon L, et al. The intestinal microbiota contributes to the ability of helminths to modulate allergic inflammation. Immunity 2015; 43: 998-1010.

48 Rabot S, Membrez M, Blancher F, et al. High fat diet drives obesity regardless the composition of gut microbiota in mice. Sci Rep 2016; 6: 32484.

49 Jakobsdottir G, Xu J, Molin G, et al. High-fat diet reduces the formation of butyrate, but increases succinate, inflammation, liver fat and cholesterol in rats, while dietary fibre counteracts these effects. PLoS One 2013; 8: e80476.

50 Chen Y, Dales R, Jiang Y. The association between obesity and asthma is stronger in nonallergic than allergic adults. Chest 2006; 130: 890-895.

51 Stein MM, Hrusch CL, Gozdz J, et al. Innate immunity and asthma risk in Amish and Hutterite farm children. N Engl J Med 2016; 375: 411-421.

52 Huang YJ, Nelson CE, Brodie EL, et al. Airway microbiota and bronchial hyperresponsiveness in patients with suboptimally controlled asthma. J Allergy Clin Immunol 2011; 127: 372-381 e371-373. 
53 Denner DR, Sangwan N, Becker JB, et al. Corticosteroid therapy and airflow obstruction influence the bronchial microbiome, which is distinct from that of bronchoalveolar lavage in asthmatic airways. J Allergy Clin Immunol 2016; 137: 1398-1405 e1393.

54 Haldar P, Pavord ID, Shaw DE, et al. Cluster analysis and clinical asthma phenotypes. Am J Respir Crit Care Med 2008; 178: 218-224.

55 Vestbo J, Hurd SS, Agusti AG, et al. Global strategy for the diagnosis, management, and prevention of chronic obstructive pulmonary disease: GOLD executive summary. Am J Respir Crit Care Med 2013; 187: $347-365$.

56 Wang Z, Bafadhel M, Haldar K, et al. Lung microbiome dynamics in COPD exacerbations. Eur Respir J 2016; 47: 1082-1092.

57 Huang YJ, Sethi S, Murphy T, et al. Airway microbiome dynamics in exacerbations of chronic obstructive pulmonary disease. J Clin Microbiol 2014; 52: 2813-2823.

58 Yadava K, Pattaroni C, Sichelstiel AK, et al. Microbiota promotes chronic pulmonary inflammation by enhancing IL-17A and autoantibodies. Am J Respir Crit Care Med 2016; 193: 975-987.

59 Molyneaux PL, Mallia P, Cox MJ, et al. Outgrowth of the bacterial airway microbiome after rhinovirus exacerbation of chronic obstructive pulmonary disease. Am J Respir Crit Care Med 2013; 188: 1224-1231.

60 Gibson RL, Burns JL, Ramsey BW. Pathophysiology and management of pulmonary infections in cystic fibrosis. Am J Respir Crit Care Med 2003; 168: 918-951.

61 Lyczak JB, Cannon CL, Pier GB. Lung infections associated with cystic fibrosis. Clin Microbiol Rev 2002; 15: $194-222$.

62 Lynch SV, Bruce KD. The cystic fibrosis airway microbiome. Cold Spring Harb Perspect Med 2013; 3: a009738.

63 Coburn B, Wang PW, Diaz Caballero J, et al. Lung microbiota across age and disease stage in cystic fibrosis. Sci Rep 2015; 5: 10241.

64 Del Porto P, Cifani N, Guarnieri S, et al. Dysfunctional CFTR alters the bactericidal activity of human macrophages against Pseudomonas aeruginosa. PLoS One 2011; 6: e19970.

65 Abman SH, Ogle JW, Harbeck RJ, et al. Early bacteriologic, immunologic, and clinical courses of young infants with cystic fibrosis identified by neonatal screening. J Pediatr 1991; 119: 211-217.

66 Armstrong DS, Grimwood K, Carlin JB, et al. Lower airway inflammation in infants and young children with cystic fibrosis. Am J Respir Crit Care Med 1997; 156: 1197-1204.

67 Mika M, Korten I, Qi W, et al. The nasal microbiota in infants with cystic fibrosis in the first year of life: a prospective cohort study. Lancet Respir Med 2016; 4: 627-635.

68 Prevaes SM, de Winter-de Groot KM, Janssens HM, et al. Development of the nasopharyngeal microbiota in infants with cystic fibrosis. Am J Respir Crit Care Med 2016; 193: 504-515.

69 Regev-Yochay G, Dagan R, Raz M, et al. Association between carriage of Streptococcus pneumoniae and Staphylococcus aureus in children. JAMA 2004; 292: 716-720.

70 van den Bergh MR, Biesbroek G, Rossen JW, et al. Associations between pathogens in the upper respiratory tract of young children: interplay between viruses and bacteria. PLoS One 2012; 7: e47711.

71 Bruscia EM, Bonfield TL. Cystic fibrosis lung immunity: the role of the macrophage. J Innate Immun 2016; 8: $550-563$.

72 Bernasconi E, Pattaroni C, Koutsokera A, et al. Airway microbiota determines innate cell inflammatory or tissue remodeling profiles in lung transplantation. Am J Respir Crit Care Med 2016; 194: 1252-1263.

73 Lipuma JJ. The changing microbial epidemiology in cystic fibrosis. Clin Microbiol Rev 2010; 23: $299-323$.

74 Reen FJ, Flynn S, Woods DF, et al. Bile signalling promotes chronic respiratory infections and antibiotic tolerance. Sci Rep 2016; 6: 29768.

75 Hartert M, Senbaklavacin O, Gohrbandt B, et al. Lung transplantation: a treatment option in end-stage lung disease. Dtsch Arztebl Int 2014; 111: 107-116.

76 Chaparro C, Maurer J, Gutierrez C, et al. Infection with Burkholderia cepacia in cystic fibrosis: outcome following lung transplantation. Am J Respir Crit Care Med 2001; 163: 43-48.

77 Alexander BD, Petzold EW, Reller LB, et al. Survival after lung transplantation of cystic fibrosis patients infected with Burkholderia cepacia complex. Am J Transplant 2008; 8: 1025-1030.

78 Dickson RP, Erb-Downward JR, Freeman CM, et al. Changes in the lung microbiome following lung transplantation include the emergence of two distinct Pseudomonas species with distinct clinical associations. PLoS One 2014; 9: e97214.

79 Hayes D Jr, Weiland A, Kirkby S, et al. Gram-negative infection and bronchiectasis in lung transplant recipients with bronchiolitis obliterans syndrome. Thorac Cardiovasc Surg 2013; 61: 240-245.

80 Song JW, Hong SB, Lim CM, et al. Acute exacerbation of idiopathic pulmonary fibrosis: incidence, risk factors and outcome. Eur Respir J 2011; 37: 356-363.

81 Tsukamoto K, Hayakawa H, Sato A, et al. Involvement of Epstein-Barr virus latent membrane protein 1 in disease progression in patients with idiopathic pulmonary fibrosis. Thorax 2000; 55: 958-961.

82 Moore BB, Moore TA. Viruses in idiopathic pulmonary fibrosis. Etiology and exacerbation. Ann Am Thorac Soc 2015; 12: Suppl. 2, S186-S192.

83 Wootton SC, Kim DS, Kondoh Y, et al. Viral infection in acute exacerbation of idiopathic pulmonary fibrosis. Am J Respir Crit Care Med 2011; 183: 1698-1702.

84 Molyneaux PL, Cox MJ, Willis-Owen SA, et al. The role of bacteria in the pathogenesis and progression of idiopathic pulmonary fibrosis. Am J Respir Crit Care Med 2014; 190: 906-913.

85 Han MK, Zhou Y, Murray S, et al. Lung microbiome and disease progression in idiopathic pulmonary fibrosis: an analysis of the COMET study. Lancet Respir Med 2014; 2: 548-556.

86 Molyneaux PL, Cox MJ, Wells AU, et al. Changes in the respiratory microbiome during acute exacerbations of idiopathic pulmonary fibrosis. Respir Res 2017; 18: 29.

87 Molyneaux PL, Willis Owen SAG, Cox MJ, et al. Host-microbial interactions in idiopathic pulmonary fibrosis. Am J Respir Crit Care Med 2017; 195: 1640-1650.

88 Huang Y, Ma SF, Espindola MS, et al. Microbes associate with host innate immune response in idiopathic pulmonary fibrosis. Am J Respir Crit Care Med 2017; 196: 208-219.

89 Cui L, Morris A, Ghedin E. The human mycobiome in health and disease. Genome Med 2013; 5: 63.

90 Wylie KM. The virome of the human respiratory tract. Clin Chest Med 2017; 38: 11-19. 
91 Jung $\mathrm{WH}$, Croll D, Cho JH, et al. Analysis of the nasal vestibule mycobiome in patients with allergic rhinitis. Mycoses 2015; 58: 167-172.

92 van Woerden HC, Gregory C, Brown R, et al. Differences in fungi present in induced sputum samples from asthma patients and non-atopic controls: a community based case control study. BMC Infect Dis 2013; 13: 69.

93 Wheeler ML, Limon JJ, Bar AS, et al. Immunological consequences of intestinal fungal dysbiosis. Cell Host Microbe 2016; 19: 865-873. 